\title{
A New Approach to Light Scattering from Nanotextured Interfaces for Silicon Thin-Film Solar Cells
}

\author{
C. Battaglia ${ }^{1}$, D. Dominé ${ }^{1,2}$, F.-J. Haug ${ }^{1}$ and C. Ballif ${ }^{1}$ \\ ${ }^{1}$ Ecole Polytechnique Fédérale de Lausanne (EPFL), Institute of Microengineering (IMT), \\ Photovoltaics and Thin Film Electronics Laboratory, 2000 Neuchâtel, Switzerland \\ ${ }^{2}$ University of Applied Sciences and Arts of Southern Switzerland (SUPSI), \\ Institute of Applied Sustainability to the Built Environment (ISAAC), 6952 Canobbio, Switzerland \\ Corresponding author e-mail: corsin.battaglia@epfl.ch,
}

\begin{abstract}
A new approach is presented to determine the angular and spectral characteristics of light diffusely scattered from nanotextured front electrodes into the absorbing silicon layer of thin-film silicon solar cell devices. OCIS codes: (040.5350) Photovoltaic
\end{abstract}

Advanced light management concepts are crucial to further improve conversion efficiencies of silicon thin-film solar cells, as the absorption coefficient of silicon is small in the near infrared region. The most common approach to improve optical performance is by means of light scattering at randomly textured interfaces. However, neither the ideal interface morphology, respecting restrictions imposed by the growth of the silicon layers, nor the ideal scattering characteristics, which maximizes the photo-generated current in the cell, have been identified to date.

We use naturally textured boron-doped $\mathrm{ZnO}$ surfaces with randomly-oriented pyramidal features with a statistical size distribution grown by low-pressure chemical vapour deposition (LP-CVD) for the development of hydrogenated microcrystalline silicon solar cells [1]. ZnO serves simultaneously as electrical front contact for the cell and as light scatterer enhancing the light path in the absorbing silicon layer.

Generally light scattering improves with increasing surface roughness, i.e. average pyramid size, of the $\mathrm{ZnO}$ front contact. However, zones of porous material, called cracks, appear in the photoactive microcrystalline layer when the roughness of the front contact exceeds a certain threshold. Previous work has demonstrated that these cracks have a detrimental effect on the fill factor $(\mathrm{FF})$ and open circuit voltage $\left(\mathrm{V}_{\mathrm{oc}}\right)$ of the cell as they act as bad diodes with a high reverse saturation current [2]. Crack density may be reduced by an appropriate plasma treatment of the $\mathrm{ZnO}$ surface, transforming the originally "V-shaped" valleys in between pyramids into "U-shaped" valleys [3]. However, such a surface treatment also decreases the light scattering capacity of the structure.
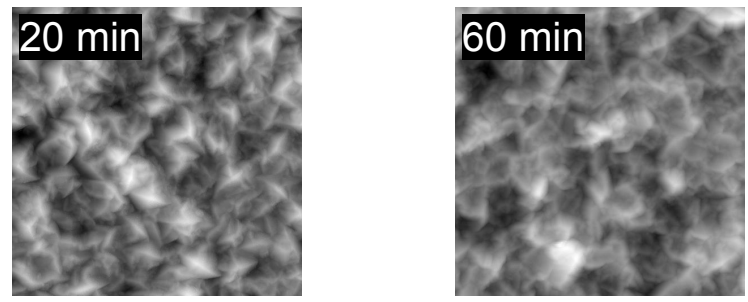

Figure 1: AFM images of LP-CVD $\mathrm{ZnO}$ with $20 \mathrm{~min}$ and $60 \mathrm{~min}$ plasma surface treatement. Image size 10x10 um².

Fig. 1 shows surface topographies obtained by atomic force microscopy (AFM) of 4.8 um thick $\mathrm{ZnO}$ layers grown on borosilicate glass. For use in microcrystalline cells, the samples in Fig. 1 were subjected to a plasma treatment of 20 and 60 minutes respectively. Samples treated only 20 min, resulting in a root mean square (rms) roughness of $150 \mathrm{~nm}$, exhibit ideal light trapping properties, but are still too rough to obtain good fill factors and open circuit voltages. Samples treated $60 \mathrm{~min}$, with a rms roughness of $120 \mathrm{~nm}$, possess an ideal morphology for obtaining good electrical properties at the expense of reduced light trapping. This can be seen directly in the external quantum efficiency (EQE) curves in Fig. 2, giving the probability of an incoming photon to be converted into an electron-hole pair within the absorbing silicon layer of the microcrystalline cell.

When studying light trapping issues in the context of silicon thin-film solar cells, one is primarily interested in the angular and spectral dependence of light diffusely scattered across the nanotextured $\mathrm{ZnO}$ surfaces into the absorbing silicon layer. However, as scattering into silicon is not directly accessible by experiment, one is restricted to perform scattering experiments into air, although scattering of light at the $\mathrm{ZnO}$-air interface differs considerably from scattering at the $\mathrm{ZnO}$-silicon interface due to the difference in the refractive index contrast $(\mathrm{n}=1$ for air, $\mathrm{n}=2$ for $\mathrm{ZnO}, \mathrm{n}=4$ for silicon). 


\section{PTuB5.pdf}

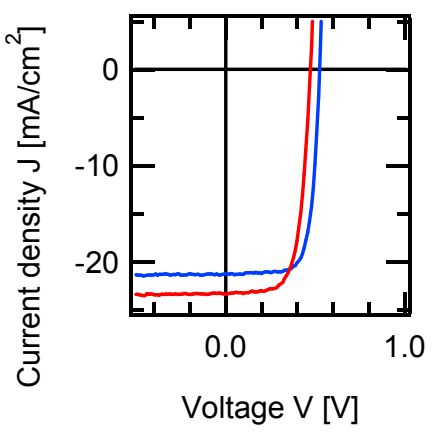

$-20 \mathrm{~min}$
$\mathrm{~V}_{\mathrm{oc}}=472 \mathrm{mV}, \mathrm{J}_{\mathrm{sc}}=23.8 \mathrm{~mA} / \mathrm{cm}^{2}$,
$\mathrm{FF}=69 \%$, Efficiency $=7.8 \%$
$-60 \mathrm{~min}$
$\mathrm{~V}_{\mathrm{oc}}=525 \mathrm{mV}, \mathrm{J}_{\mathrm{sc}}=21.2 \mathrm{~mA} / \mathrm{cm}^{2}$,
$F F=74 \%$, Efficiency $=8.2 \%$

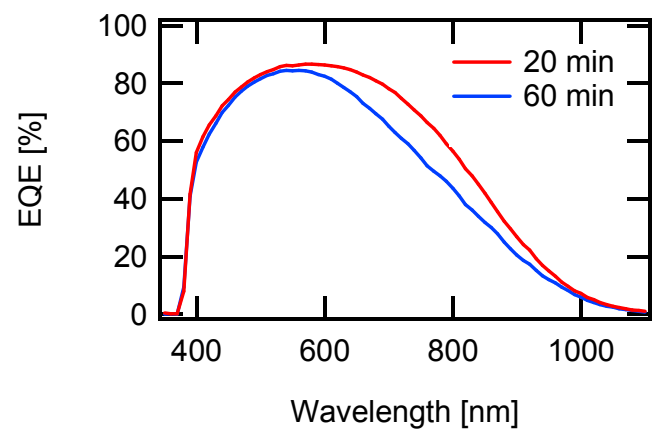

Figure 2: Current-voltage characteristics and external quantum efficiency (EQE) for microcrystalline silicon thin-film solar cells on 20 min and 60 min plasma-surface-treated LP-CVD ZnO.

We recently reported a new computational approach to investigate the angular and spectral dependence of light diffusely scattered across nanotextured interfaces [4]. Our model makes use of a slightly modified RayleighSommerfeld diffraction integral and requires only measured surface profile data and the refractive index as input. Our treatment does not require the usual restriction that the structure size of the texture should be much smaller than the wavelength. The scattered light intensity at the point of observation is obtained by superimposing spherical waves emitted from the scattering surface, taking into account the phase shifts that light accumulates on its passage trough the measured profile.

To validate our approach, we performed calculations for scattering into air. Fig. 3 presents the comparison between experimental and calculated angle-resolved scattering (ARS) curves measured for the two $\mathrm{ZnO}$ surfaces at a wavelength of $543 \mathrm{~nm}$. The agreement between experiment and calculation is excellent for angles below $50^{\circ}$ (paraxial approximation involved). As can be seen, the $20 \mathrm{~min}$ treated samples exhibits more pronounced scattering into higher angles, which is beneficial for enhancing the current density of the cell. The oscillations in the calculations are an artefact due to the limited AFM image size and may be reduced by using larger images.
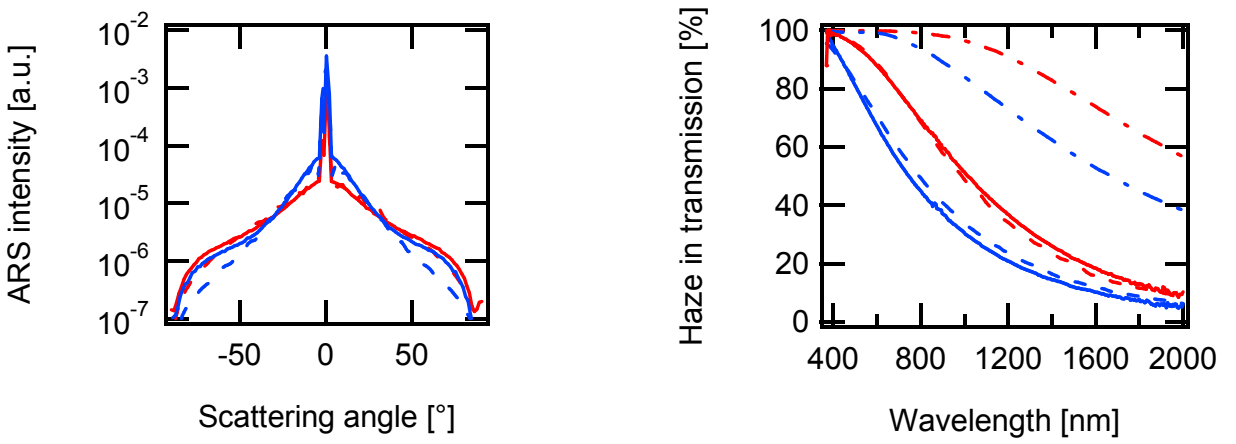

Scattering into air:

Experiment

$20 \mathrm{~min}$

$60 \mathrm{~min}$

Theory

- - $20 \mathrm{~min}$

$--.60 \mathrm{~min}$

Scattering into silicon:

Theory

$-\cdots 20 \mathrm{~min}$

-..60 min

Figure 3: Angular and spectral dependence of light scattered on nanotextured LP-CVD ZnO with 20 min and 60 min plasma surface treatement.

In Fig. 3 we also show the spectral dependence of the scattered light. Experimentally the haze in transmission is determined by taking the ratio between diffuse and total transmittance. Theoretically we can derive the haze value at a given wavelength by integrating the calculated ARS intensity without the spectral peak at 0 degrees and normalizing it by the total scattered intensity. The calculated curves agree with the experimental curves.

We finally present the results for the calculations for scattering into silicon, which behave quite different from scattering into air. As can be seen in Fig. 3) the haze at low wavelength, is close to $100 \%$ for both, the 20 min and 60 min treated surface. When going towards larger wavelength, where light trapping becomes important, the haze for the 60 min treated surface drops faster than for the 20 min treated surface.

[1] S. Fay, J. Steinhauser, N. Oliveira, E. Vallat-Sauvain and C. Ballif, Thin Solid Films 515, 8559 (2007)

[2] M. Python, E. Vallat-Sauvain, J. Bailat, D. Domine, L. Fesquet, A. Shah, and C. Ballif, J. Non-Crystalline Solids 354, 2258 (2008)

[3] D. Domine, P. Buehlmann, J. Bailat, A. Billet, A. Feltrin and C. Ballif, Phys. Stat. Solidi (RRL) 2, 163 (2008)

[4] D. Domine, F.-J. Haug, C. Battaglia, and C. Ballif, accepted for publication in J. Appl. Phys. (2010) 